FRANCESCO D'ERRICO

\title{
FUOR DI METAFORA
}

SETTE OSSERVAZIONI

SULL'IMPROVVISAZIONE MUSICALE

\author{
prefazione \\ Paolo de Vita \\ postfazione \\ Mauro Maldonato
}

Editoriale Scientifica

Napoli 
Tutti i diritti sono riservati

(C) 2015 Editoriale Scientifica srl

Via San Biagio dei Librai 39 80138 Napoli

www.editorialescientifica.com

info@editorialescientifica.com

ISBN 978-88-6342-826-1 


\section{Indice}

7 Nota editoriale: dentro e fuori l'ossimòro di Luigi Maria Sicca

17 Prefazione di Paolo de Vita

35 Premessa

41 Prima osservazione

51 Seconda osservazione

63 Terza osservazione

75 Quarta osservazione

87 Quinta osservazione

99 Sesta osservazione

113 Settima osservazione

123 Conclusioni

127 Postfazione di Mauro Maldonato

143 Bibliografia essenziale

149 Indice dei nomi

151 Hanno scritto nella Collana punto org 



\section{Nota editoriale: dentro e fuori l'ossimòro}

\section{Luigi Maria Sicca}

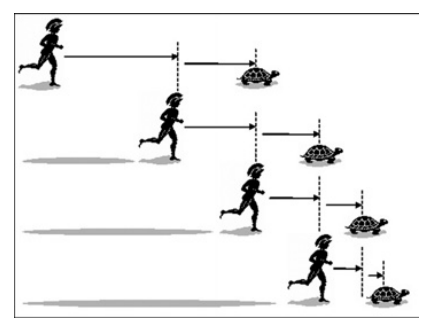

L'incontro accademico con Francesco D'Errico ha inizio nel 2010, con una serie di esperimenti a prevalente valenza didattica. Invitai l'autore di questo libro in aula a uno dei miei corsi di Organizzazione Aziendale, nell'ambito di un ciclo pluriennale dal titolo: "Capacità di ascolto e Organizzazione Aziendale. Musicisti e manager a confronto"1 ${ }^{1}$. Coinvolsi eterogenei professionisti. Ciascun ospite era portatore di storie scolari e di apprendimento idiosincratiche, con strutture di conoscenza situate; ciascuno, portatore di modi unici e originali di leggere e scrivere l'azione organizzativa: musicisti, da un lato, manager o imprenditori, dall'altro $^{2}$. Consapevole che musicista può significare molte cose, tante, almeno quanto imprenditore o manager. Il contributo di D'Errico e del suo trio si collocò, inizialmente, in questa cornice e si focalizzò, come naturale che fosse, dovendo maneggiare il jazz, sia su esperienze di team buiding sia su quelle di team working. Ne venne fuori una pubblicazione (Sicca, 2013) che vide anche la presenza di Francesco.

Il 25 settembre 2013, in occasione di un seminario di presentazione di quella ricerca con musicisti e manager presso l'Università Bocconi, Francesco D'Errico illustrò il suo contri-

${ }^{1}$ A quel progetto parteciparono, tra gli altri: Massimo Marrelli, Paola Giampaolo, Francesco Vizioli, Francesco D. Perillo, Renato Viscardi, Marco Vitali, Chiara Mallozzi, Davide Bizjak, Maria Vittoria Colucci d'Amato, Raffaele Cercola, Alain Meunier, Enzo Rullani.

2 Redazione online Corriere del Mezzogiorno (2010). 
buto alla partita, ponendo l'attenzione su due aspetti che il lettore ritroverà nelle prossime pagine: la cessione di potere e l'accoglienza della vulnerabilità. Due risorse per declinare l'improvvisazione fuor di metafora: l'improvvisazione come archetipo di specifiche pratiche professionali (quelle, per esempio, del concertista), per poi accadere davvero, in numerosi contesti di azione organizzativa, ben oltre le esperienze del jazz o della musica in generale ${ }^{3}$.

L'iniziale esperienza didattica e di ricerca, in realtà, aveva alle spalle un articolato percorso che mi accompagnava, già allora, da diversi anni ${ }^{4}$ : la domanda di fondo riguardava, con specifico riferimento agli studi organizzativi ed economico-manageriali, come intersecare linguaggi di differente scaturigine dottrinale. In ciò, eravamo consapevoli di affrontare, seppur limitatamente al nostro contesto, un ampissimo problema teorico, critico, peraltro, nella storia dei saperi umani e sociali. Proprio perché centrati sul linguaggio, sapevamo di avere a che fare con un tema trasversale a tutte le scienze. A me pareva, già allora, che per comprendere in profondità il senso di un'intersezione così delicata, dovesse essere svolto un massiccio lavoro di ricerca empirica, con il supporto e la padronanza di specifiche competenze grammaticali: in questo caso, attraverso un viaggio, gomito a gomito, economisti, manager e musicisti. Possibile ri-

${ }^{3} \mathrm{Fu}$ quella l'occasione per avviare un percorso di ricerca, finalizzato a verificare il significato dell'improvvisazione entro quattro domìni: la gastronomia, la musica, la didattica e la chirurgia generale e d'urgenza. Per ciascuno di essi, abbiamo costruito una track list (validata da un intervistatopilota) e sottoposta a un numero omogeneo di informatori. Un primo esito di quel lavoro lo presentammo al $30^{\circ}$ EGOS Colloquium, che si tenne presso la Rotterdam School of Management, Erasmus University, dal 3 al 5 luglio 2014.

${ }^{4} \mathrm{Ci}$ sia consentito di rinviare a Sicca (2012). In quel volume, articolavo i principali temi che toccai in occasione della lezione che tenni come prolusione per l'inaugurazione dell'a.a. 2010-2011 presso l'Università del Sannio, Conservatorio Statale di Musica di Benevento Nicola Sala. 
sposta per evitare facili scivoloni semantici e, di conseguenza, in termini di metodologia della ricerca.

$$
* * *
$$

Fuor di metafora. Sette osservazioni sull'improvvisazione musicale partecipa alla più ampia riflessione in seno a puntOorg sugli assunti, mai scontati o acquisiti semel et in perpetuum, che orientano i possibili modi di stare insieme. Su questo, noi riteniamo sia urgente interrogarsi, a partire da un ripensamento della storica, quanto attuale, questione della pressione sui confini: è la sfida che sta attraversando l'Europa in questo secondo decennio di millennio. A essa siamo chiamati, cercando domande o risposte, ciascuno di noi con la propria cassetta degli attrezzi da mettere in campo. È una sfida etica, perché radicata, innanzitutto, su libere scelte epistemologiche, attraverso le quali sarà possibile, forse, introdurre soluzioni politiche, riconoscibili nella realtà di tutti i giorni, dei popoli, delle nazioni, delle nostre economie.

Questo nuovo libro articola l'iniziale ipotesi: partire dalle premesse di cui ciascuno è portatore, per guardare fuori la finestra del proprio àmbito di provenienza. Ciò allo scopo di comprendere dinamiche meno evidenti (eppure, presenti con la propria strutturazione paradossale) nelle organizzazioni maggiormente studiate dalla letteratura mainstream ${ }^{5}$. Ovvero, per comprendere le logiche sottostanti la competizione contemporanea, in uno scenario caratterizzato da un crisi lunga e sottopercepita, forse di sistema. Cercando domande, al cospetto di un'identità inquieta e in movimento della comunità (e di un'economia) europea ancora tanto giovane: è questo uno dei temi ricorrenti del progetto puntOorg, lanciato con una antologia sui saperi umanistici quali fonti di sapere manageriale (Sicca, 2010) e giunto, con questo nuovo contributo, al volume numero 16.

${ }^{5}$ Su questo punto, ci sia consentito di rinviare a Sicca $(1997 ; 2000 ; 2001)$. 
Ci stiamo lavorando da tempo. La questione è stata sollevata più volte, già in precedenti numeri della Collana: imparare a stare, diversamente, insieme è, a nostro avviso, il nodo da sciogliere per fronteggiare con coraggio il susseguirsi (in settanta anni di pace ufficiale nell'Occidente industrializzato) di crisi continue e con intervalli drammaticamente asintotici tra una grande crisi e la successiva, forse ancora più grande, di certo più lunga. Stare insieme impone una consapevolezza del perimetro entro cui interagire: se è vero che l'identità si staglia per differenza tra ciò che è dentro e ciò che è fuori, è proprio la percezione dei confini di uno spazio abitato che decide la partita della convivenza. L'improvvisazione, così come trattata in questo volume, ha molto da insegnare in proposito!

Nessun confine è dato ex ante. Lo insegna la Storia, la Politica, lo insegnano gli studi organizzativi (de Vita, 2007). Il concetto di azione (e, quindi, di attore) infrange la certezza delle pareti e ruba la scena alla più rassicurante e statica organizzazione, specie di fronte al bisogno o desiderio, di tempo in tempo, in questo tempo non da meno, di percorrere le frastagliate vie del cambiamento, mai scevre di ostacoli, di resistenze, tanto fisiologiche quanto inevitabili, da riconoscere e affrontare:

Bei tempi quando i confini erano linee, tracciati netti ed evidenti che separavano senza ambiguità: di qua o di là, ognuno al suo posto. Che si trasformassero in frontiere, attorno alle quali si accendevano contese che portavano spesso a "fare-fronte" e dunque a combattere, era solo una ragione in più per presidiarli accuratamente, tamponando ogni falla e blindando i passaggi.

Così che, quando non scoppiavano scaramucce o vere proprie guerre, quello era lo spazio privilegiato dei contrabbandieri, uomini dedicati al rischio dell'attraversamento furtivo e dei commerci irregolari con il "nemico".

Erano dunque tempi di sicurezze esemplari e delle appartenenze granitiche, sostenute dalla dottrina accreditata e dalla voglia di dare per scon- 
tato che, almeno in un punto definito del territorio, non era lecito dubitare. Lì finiva un mondo; al di là era terra straniera.

Poi le cose, poco a poco, si sono andate confondendo.

Sono caduti i grandi sistemi ideologici che facevano da supporto alle logiche di separazione, si sono allargati $i$ mercati in dimensioni mondiali, $i$ sistemi di comunicazione hanno ridotto le distanze e le stesse guerrelocali formalmente, ma globali nella percezione e nella visualizzazione mediatica - hanno reso evidente che tracciare confini era impresa quasi impossibile dal momento che le linee che definivano "il noi" e "gli altri" erano sempre più mobili, intrecciate, labili. ${ }^{6}$

Ripensare e, diversamente, agire piani identitari così profondi, a propria volta di confine, non è certo cosa da poco. Richiede saggezza, età, pensiero. A volte, forse, vecchiezza. Richiede, insomma, come tanta letteratura ci dice con forza, avere familiarità con gli archetìi, primi esemplari assoluti, modelli, secondo il Devoto-Oli (Devoto e Oli, 1971); secondo noi, potente strumento, occasione vincente per una gestione flessibile, quanto meno realistica, se non duratura, del cambiamento e dell'innovazione. È questo l'approccio all'improvvisazione che proponiamo.

Quando si attraversa la strada, la vista di un camion verso di noi stimola, nella maggior parte dei casi, una reazione immediata e, presumibilmente, prudente. Non ci fermiamo per chiedere a che velocità stia correndo il camion, da dove giunga, quanto spesso ciò accada oppure quali le intenzioni del guidatore. Semplicemente, ci muoviamo. Il nostro studio sul camion riguarda poco più che un rapido sguardo, che svela alla maggior parte di noi un significato simbolico del potere della velocità. Perciò, mettiamo in atto una risposta spontanea e immediata, quasi in modo obbligato. Questo è l'obiettivo delle ricerche qua-

${ }^{6}$ Celli (2014, p. 89). 
litative: identificare quei simboli e il loro significato, allo scopo di registrare un modello di risposte che questi simboli suscitano e provocano in noi.

Questo esempio (van Maanen, 1979) solleva la questione metodologica relativa alla definizione dei confini della ricerca empirica di stampo qualitativo. Questione che a noi pare direttamente collegata al discorso sull'improvvisazione, così come affrontato da D'Errico. Perché quell'esempio suggerisce il nesso tra segno e significato, in funzione di una struttura interpretativa: quando questa è accettata, gli strumenti di analisi trovano agevolmente una rigorosa collocazione, consentendo di far bene ricerca. In tal senso, i dati che raccogliamo nella vita di tutti i giorni sono dello stesso tipo di quelli che un ricercatore qualitativo prova a raccogliere e registrare: sono simbolici, radicati nel contesto, criptici e riflessivi (se non vengono indagati) e la loro leggibilità e semplificazione dipende dal significato e dall'interpretazione che se ne riesce a dare. Da cui, l'importanza di tradurre i fenomeni in linguaggio simbolico, riducendo così le distanze tra ciò che è indicato e gli indicatori, fra teorie e fatti, tra contesto e azione.

È forse questa la funzione del jazz e, più in generale, dell'improvvisazione dentro e fuori i linguaggi della musica? È forse questa la proposta con cui D'Errico trascende il particolare, portando le Sette osservazioni a fonte di sapere per le scienze umane e sociali e, nello specifico, a fonte di sapere economicomanageriale?

Le risposte possibili probabilmente dipendono, ancora una volta, dai modi con cui si gestiscono i confini: saper dove mettere le virgolette, per riprendere ancora il lessico di van Maanen, non è cosa facile. Significa sapersi muovere (con la stessa destrezza di chi ha di fronte un camion in corsa) entro uno spazio teorico ed epistemologico pluralistico. Significa, in ultima analisi, sapersi muovere tra riduzionismo e costruttivismo, saper contemperare il trade off (sollevato da D'Errico) tra modello e slancio ideale 
(Manara, 2001), fra regolarità e apprezzamento dell'irregolarità, tra la certezza degli occhiali dei nostri framework teorici e la possibilità di leggere altro, in modo anche sfocato. Ogni sguardo sul mondo lo è, perché variabile: vera e impalpabile è la sensibilità dell'occhio, funzione della lunghezza d'onda della luce percepita da ognuno. L'improvvisazione proposta da D'Errico questo lo sa: sa che il modello orienta in funzione di premesse e assunti; lo slancio ideale spinge verso le sperimentazioni, sentieri spesso inediti. Il secondo non esclude il naufragio, il fallimento; il primo non esclude la gabbia.

Questa idea di improvvisazione fuor di metafora, ma anche metodo, chiave di lettura per diagnosticare la realtà in movimento, non è per certi versi nuova alla letteratura economica e organizzativa. È, invece, punta dell'iceberg di un articolato percorso su prestigiose riviste internazionali già dalla metà degli anni Settanta, con una crescente attenzione assegnata alle esperienze emozionali nei processi di decision making (Sicca, 2012; Maldonato, 2015). Mintzberg $(1973,1976)$ riconosceva un ruolo paritario a entrambi gli emisferi del sistema nervoso centrale, destro e sinistro, nella costruzione delle decisioni. Io penso che quell'approccio, su cui le neuroscienze dichiarano di avere ancora moltissimo da lavorare, possa essere liberamente associato a un altro movimento di pensiero, culminato nell'assegnazione, nel 1978, del premio Nobel a Herbert A. Simon "per le sue pionieristiche ricerche sul processo decisionale nelle organizzazioni economiche". Un contributo (Simon, 1958 [1947]) che, irrompendo nella tradizione del pensiero neoclassico, introduce il concetto di bounded rationality, centrale per la comprensione dell'azione manageriale guidata da limiti cognitivi e da un ammontare finito di risorse in presenza di costi associati a contratti incompleti.

Se si fossero analizzate con freddezza le forze in gioco non sarebbe stato difficile prevedere come si sarebbe snodato il caso greco. [...] Non si può 
permettere che la più innovativa istituzione mondiale degli ultimi cento anni finisca vittima di un populismo alimentato da un crescente senso di lontananza, di impotenza e di mancanza di solidarietà tra le istituzioni europee. $^{7}$

Quali ponti uniscono e separano la riflessione di Politica Internazionale proposta da uno dei fondatori dell'odierna Europa e le osservazioni filosofiche sull'improvvisazione proposte da Francesco D'Errico in questo nuovo volume della Collana punto org? Perché, in altri termini, connettere l'urgenza di approfondire le gravi ragioni della crisi di prestigio che investe l'EuropaIstituzione con una proposta teorica che viene da un ricercatore, musicista, filosofo?

Sono proprio le dicotomie, che accompagneranno il nostro lettore, a fornirci possibili risposte: potere $e$ vulnerabilità; razionale $e$ non razionale; dentro $e$ fuori; modello $e$ slancio ideale; ricerca quantitativa $e$ ricerca qualitativa; organizzazione $e$ azione; significato $e$ senso; prossimale $e$ distale. Il lettore potrà scorrere le pagine scritte da Francesco cogliendo queste co-esistenze a più livelli, ben oltre un ossimòro ipoteticamente sterile. Nel segno dei paradossi della millenaria tradizione, quelli fertili, scarsamente soddisfatti delle risposte, certo possibili, offerte dal mondo moderno. È questo, a mio avviso, uno degli insegnamenti ricavabili dalla lezione sull'improvvisazione che qui proponiamo: la sfida è politica, sia essa di Politica Economica e Industriale, di Politica Culturale o per l'Istruzione (e la formazione) delle future generazioni di cittadini europei ${ }^{8}$, chiamati a confrontarsi, da un lato, con le esigenze di competitività poste dagli scenari geopolitici; dall'altro, con differenti modelli di solidarietà inter-istituzionale.

${ }^{7}$ Prodi (2015).

${ }^{8}$ Si rinvia al recentissimo manuale (il numero 15 in Collana) di Francesco Piro (2015). 
L'improvvisazione, dunque, luogo dell'imperfezione, rappresentazione del fisiologico ibrido che la realtà ci dona, in tutta la sua pienezza.

$$
* * *
$$

Le ricerche puntOorg sono sempre il risultato di un gioco di squadra, che funziona grazie a specifiche ed eterogenee competenze che, messe in campo al servizio della comunità, consentono un risultato a più voci, comunque corale e, talvolta, volutamente dispari.

Questa volta, il solista è Francesco D'Errico, accompagnato da Paolo de Vita e Mauro Maldonato. Con nitore, partono dalla propria angolazione teorico-disciplinare, per tematizzare l'improvvisazione. La prefazione, dunque, espone il punto di vista degli studi organizzativi; la postfazione sussume la ricerca in campo neuroscientifico. A Paolo e a Mauro va la nostra gratitudine per avere accolto la sfida, arricchendo così le prospettive in seno a puntOorg. Grazie a Ernesto Tatafiore per avere realizzato la copertina del libro, i cui disegni interni (sette, uno per ciascuna osservazione) ne sono particolari, dando in questo modo continuità e, al tempo stesso, ampliando con la sua arte il gesto infantile che, da sempre, caratterizza la linea grafica della Collana. Grazie a Antonio Fruttaldo, per l'intelligente e cólto impegno profuso sulle bozze.

Kos, Agosto 2015

Napoli, 19 settembre 2015 Università degli Studi Federico II 